\title{
Der Übersetzungssektor in Krisenzeiten in Spanien und die Erscheinung der neuen Technologien. Die neuen Übersetzung-Apps für Android-Smartphones
}

\author{
https://dx.doi.org/10.12795/futhark.2014.i9.11 \\ Concepción Mira Rueda \\ Universidad de Málaga \\ mirarc@uma.es
}

\begin{abstract}
During the last few years, the financial crisis in Europe and other countries in the world has had a profound impact on every professional branch. Among these, we highlight its negative influence on the translation and interpreting branch. In this paper, we will tackle three main questions. Firstly, we will analyse the way labour market for translators and interpreters has been undermined by the crisis. Subsequently, the decisive factors will be explained. In addition, we will see how the financial crisis has become a matter of particular concern to more and more Translation scholars. Secondly, we will deal with new technologies that have emerged and become a successful product in the midst of the current economical crisis. However, we will not deal with new technologies on the whole, but those that have to do with Translation, i.e. new apps for Android-smartphones. We will see how sophisticated are they and the way, sometimes, are they used in order to satisfy punctual translation needs on the part of individuals and enterprises, who may consider professional translations and interpreting like a luxury that not everyone can afford. Next, we will compare the latest translation apps: automatic translators and voice translators. Lastly, we will focus on how the crisis is influencing on the Spanish Universities in terms of Translation and Inter-
\end{abstract}


preting studies. We will contrast the number of student enrolments and what is the situation (reasons, goals and motivations) of the students who enrolled Translation and Interpreting studies.

Key Words: Translation, Interpreting, Technology, Profession, Teaching, Crisis, smartphone apps, automatic translator, voice translator

Zusammenfassung: In den letzten Jahren hat die finanzielle Krise in Europa und in mehreren Länder der Welt eine tiefgreifende Auswirkung auf jeder Berufsbranche gehabt. Unter diesen Berufe werden wir seinen negativen Einfluss auf den Übersetzungs- und Dolmetscherbranche hervorheben. In diesem Beitrag werden wir drei wichtigste Fragen in Angriff nehmen. Erstens werden wir den Arbeitsmarkt für Übersetzer und Dolmetscher, der durch die Krise beeinträchtigt wird, analysieren. Danach werden die entscheidenden Faktoren erkärt. Darüber hinaus werden wir sehen, wie die Finanzielle Krise ein spezielles Anliegen der Übersetzung und Dolmetschen-Wissenschaftler zurzeit ist. Zweitens werden wir neuen Technologien, die in der derzeitigen Finanzkrise ein sehr erfolgreiches Produkt geworden ist, behandeln. Allerdings werden wir nicht allgemeinen Technologien behandeln, sondern diese die mit Übersetzung und Dolmetschen zu tun haben, wie zum Beispiel die neuen Apps für Android Smartphones. Wir werden auch wie ausgeklügelt diese neuen Apps sind sehen. Wir werden ebenfalls kennen, wie häufig ist die Verwendung dieser Apps um punktuelle Übersetzungsbedürfnisse von Personen und Unternehmen zu befriedigen, hauptsächlich weil ist es leider üblich zu glauben, dass professionelle Übersetzung und Dolmetschen ein Luxus, dass sich niemand gestatten kann abzuwarten. Als nächstes werden wir die neuen Übersetzung Apps vergleichen: automatischen Übersetzer und Übersetzer mit Sprachausgabe. Schließlich werden wir uns auf die Art, in die die Krise die spanischen Universitäten in Bezug auf das Studium von Übersetzung und Dolmetschen beeinflusst hat, konzentrieren. Zum Schluss werden wir über die Zahl der immatrikulierten Studenten und die Lage der neuen "Übersetzung und Dolmetschen" Studenten (Gründe, Ziele und Motivationen) sprechen.

Schlüsselworte: Übersetzung, Dolmetschen, Technologie, Beruf, Lehre, Krise, Smartphones, Apps, automatischen Übersetzer, Übersetzer mit Sprachausgabe 


\section{EINLEITUNG}

In den letzten Jahren leidet Spanien unter den Folgen der Krise. Aus diesem Grund sind viele Unternehmen in den Bankrott getrieben worden. Heutzutage schließen die Firmen leider häufig. Letzten Endes ist die gegenwärtige Wirtschaftslage nicht gerade viel versprechend. Das hat ausgelöst, dass Unternehmen ihre Übersetzungsdienste oft eingestellt haben. Mehrmals sind es die Firmen selbst, die ihre eigenen Übersetzungen machen. Das heißt, dass sie auf eigene Rechnung übersetzen und dolmetschen. Im Klartext: Sie übersetzen ohne professionelle Hilfe.

Eine anderere Ursache, die die Arbeit von Übersetzern und Dolmetschern bedroht, ist das zunehmende Erscheinen von Software, die immer leistungsfähiger wird, wie beispielsweise die automatischen Übersetzer und die Übersetzer mit Sprachausgabe.

Die Wirtschaftskrise hat auch Einfluss auf den Übersetzungsunterricht: das Studentenprofil ist nun noch bunter als zuvor, da der Mangel an Arbeitsplätzen bewirkt hat, dass die Zahl der immatrikulierten Studenten gestiegen ist, die die Zeit zur Weiterbildung nutzen, aber nicht unbedingt am konkreten Studium interessiert sind. Das bedeutet, dass es neben den motivierten Studenten auch viele unmotivierte gibt. Dies kann Misserfolge auslösen, was eine große Verantwortung für die Dozenten eines Studienzweiges darstellt, der zu einem Beruf mit langer Tradition ausbildet. Die Übersetzungswissenschaft hat bereits seit langer Zeit ihre Bedeutung gezeigt, indem sie Sprach- und Kulturmittler für sehr unterschiedliche Zweige wie Wirtschaft, Wissenschaft, Recht, u.v.a.m. ausbildet.

Mit meinem Beitrag möchte ich zeigen, wie die Krise das Studium und die Arbeit der Übersetzungsbranche beeinflusst hat:

1. Die Forschung der aktuellen Wirtschaftszweige in Spanien, damit man die Wachtumsbranche finden kann.

2. Die Recherche über die neuen automatischen Übersetzer und die aktuellen Übersetzer mit Sprachausgabe, die für Android über Samsung Apps und Google Play erhältlich sind. 
3. Der Einfluss der Krise auf den Übersetzungs- und $D_{0}$. metschunterricht, speziell für das Sprachenpaar Spanisch-Deutsch.

Diese Untersuchung zeigt die steigende Bedeutung der Übersetzungswissenschaft in der aktuellen Welt. In Spanien ist mo. mentan der umsatzstärkste Unternehmensbereich das Mobiltelefon. Spanien ist das europäische Land, in dem trotz der Krise die meisten Smartphones gekauft werden. Meine Untersuchung zeigt, dass trotz der steigenden Zahl an automatischen Übersetzern und Übersetzer mit Sprachausgabe der menschliche Übersetzer immer noch nicht ersetzbar ist.

\section{DER ÜBERSETZUNGSEKTOR UND DIE KRISE}

Dieser Beitrag befasst sich mit der Übersetzung in Krisenzeiten. Zuerst wird die aktuelle Lage des Übersetzungssektors behandelt haben. Es gibt noch keine Übereinstimmung hinsichtlich des Datums der Ursprung der Finanzkrise. Einige Autoren sind der Meinung, dass die Krise 2007/8 mit der Spekulationsblase im Immobiliensektor begonnen hat. Manche Autoren hingegen behaupten, dass die aktuelle spanische Finanzkrise schwierig zu datieren ist, weil sie eine Frage der jahrelangen Verschwendung und der Misswirtschaft der Politiker ist. In der Tat erfahren wir noch heutzutage ihre Folgen, unddie Krise ist leider noch ein aktuelles Thema. Jeden Tag sehen (und kennen) wir Menschen, die ihren Arbeitsplatz verlieren und andere, die ihre Unternehmen schließen müssen.

In Hinsicht auf den Übersetzungssektor gibt es immer mehr Autoren, die über diese Erscheinung zahlreichen Beträge schreiben, wie beispielsweise Nogueira (2003), García Luque (2010) Davico (2013) und Grelier (2013).

Im Jahr 2010 schrieb García Luque einen Beitrag über das Wort "Krise" in den Medien und erstellte eine terminologische Analyse für Lehre und Praxis der sozioökonomischen Übersetzung für das Sprachenpaar Französisch-Spanisch. 
Gemäß García Luque ist das Wort "crisis" (Krise) heutzutage eines der am meisten gebrauchten Worte. In dieser Hinsicht betont sie:

"Si tuviéramos acceso a una muestra de los periodicos más representa-
tivos de todos los países del mundo, de los informativos más relevan-
tes o incluso si pudiésemos oír las conversaciones de sobremesa de
muchas familias de los cinco continentes, probablemente una de las pa-
labras que más se repetiría sería la palabra crisis, que se ha vuelto a co-
lar en nuestro vocabulario cotidiano desde aproximadamente la se-
gunda mitad del año 2008 como una realidad a la que no escapa casi
ningún aspecto de nuestra vida diaria."

Im Folgenden erinnern wir uns an ein Interview, das Ana Hermida (2013), Dozentin an der Universidad Autònoma de Barcelona (UAB in einer spanischen Übersetzerzeitschrift, La linterna del Traductor, gab, wo sie erklärte:

„En la actualidad, la crisis está afectando a todos los sectores, incluidas la traducción y la interpretación. Muchos traductores autónomos han tenido que buscarse otras vías para conseguir ingresos o aceptar las tarifas que se pagaban hace 10 años."

Diese Erklärungen zeigen, dass zahlreiche freiberufliche Übersetzer wegen der Krise in Spanien unter Tarif bezahlt werden. In der Gegenwart werden Tarife akzeptiert, die vor 10 Jahren bezahlt wurden. Andere Autoren sind der gleichen Meinung. Am 21. November 2012 schrieb Davico, ein italienischer Übersetzer, einen Beitrag, wo er über die niedrigen Tarife sprach. Da gab es eine Übersetzerin, Gabriella Gentile, die Davicos Beitrag kommentierte, und ihr Kommentar fand Davico so interessant, dass er einen neuen Beitrag schrieb (2013), diesmal über den Arbeitsmarkt der Übersetzung. Er fragte sich, ob der Übersetzungsarbeitsmarkt sich in einer Krise- oder Dekadenzlage befinde. Davico gelangt zu folgendem Schluss:

„Chi vive di questa professione deve mantenere un livello qualitativo alto offrendo servizi riservati a clienti che possono permettersi di pagarli. Le agenzie e i clienti giusti che pagano il giusto esistono, ma è necessario che diventino la regola e non l'eccezione."

Der Autor erklärte, dass Übersetzer ein qualitatives Niveau halten müssten. Mit anderen Worten müssen Übersetzer eine Dienstleistung 
von hoher Qualität für die Kunden bieten. Er fügt schließlich sogar hinzu, dass, obwohl es seriöse Firmen und Kunden gibt, die adäquate Tarife bezahlen, sie immer noch die Ausnahme sind, obwohl sie die Regel darstellen sollten. Andere Autoren, wie Grelier (2013) und Nogueira (2003) sind auch der gleichen Meinung. Grelier vertritt die Ansicht, dass der Übersetzungssektor sich sogar in der ganzen Eu. ropäischen Union in einer Krise befindet aufgrund der maschinellen Übersetzung. Derzeit besteht die Befürchtung, dass das gleiche mit literarischen Texten geschehen werde. Deshalb könnten diese Texte ihre Identität verlieren. Greliers Beitrag pflichtet den Worten der Direktorin der Europäischen Gesellschaft der Autoren (EGA), Camille de Toledo, bei, die hervorhebt:

"Il y a une distinction fondamentale entre d'une part la traduction communicante, propre à la pratique de l'UE, qui est à terme, "lulle affaire de machines", et d'autre part la traduction litteraire, qui est au contraire une "affaire d hommes et de femmines"."

Vor allen diesen Autoren gab es jedoch einen, der all dies vorhergesehen hat, Nogueira, einbrasilianischer Übersetzer, der 2003 über die Krise und die Schwierigkeiten des Übersetzungsarbeitsmarktes geschrieben hat: die Globalisierung, die Tarife und vor allem die hohen Anforderungen in Bezug auf den Beruf der Übersetzer und der Dolmetscher. In seinem Beitrag erklärte er, dass das Hauptproblem in Zusammenhang mit der Finanzkrise die Rezession ist. Deshalb behauptet er:

"As I see it, the most important factor in the present crisis is the recession, and there is very little we can do about it. Of course, we can become more efficient and increase our marketing effort, but, until the economy rebounds, it will be a zero-sum game: the client you gain is the client somebody else loses. Fortunately, all recessions eventually end and this will be no exception."

Die Krise hat natürlich den Beruf der Übersetzer beeinflusst, wie wir gerade gesehen haben. Für jene, die bei einem Übersetzungsunternehmen arbeiten, kann das Arbeitsvolumen niedriger sein als früher. Die Mehrheit der Übersetzer, die ihre Arbeitsplätze verloren haben, entscheidet selbständig zu arbeiten. Tatsächlich ist der Umsatz der neuen selbständigen Erwerbstätigen seit dem Jahr 2009 gestiegen, 
so die spanische Wirtschaftszeitung Cincodías (2013). Die Autonomen Regionen in Spanien, wo es den höchsten Zuwachs der Selbständigen gibt, sind: die Balearen (7,8\%, bzw. 5.984 Unternehmer) gefolgt von Andalusien (1,1\%, das heißt, 4.880 Unternehmer). Angesichts dieser Situation betracjtem die Unternehmen die Übersetzung und das Dolmetschen als Nebentätigkeit. Wegen der Krise erledigen die Unternehmen ihre Übersetzungen selbst, weil sie sparen müssen. Diese "neuen" Übersetzer sind aber Angestellte dieser Unternehmen, die zum Beispiel Englischkenntnisse haben. Sie glauben, dass sie mit anderen internationalen Kollegen in Englisch sprechen könnenund sie deshalb keinen professionellen Dolmetschdienst benötigen. Diese Praxis kann sehr gefährlich sein, denn die Sprachkenntnisse sind häufig nicht so fortgeschritten wie die Menschen angenommen hatten. Tatsache ist, dass die Folge Missverständnisse und Meinungsverschiedenheiten sein können. Das gilt auch für die Übersetzung. Die Unternehmen können sich keine professionellen Übersetzer erlauben. Also, entscheiden sie ohne professionelle Hilfe zu übersetzen. So heißt das, dass diese Übersetzungen eine mindere Qualität haben können. Häufig setzen sie Software, Apps, Webseiten, automatische Übersetzer, usw. ein, um ihre Übersetzung zu machen.

Die Frage der Verwendung der Technologie für das Übersetzen ist ein ausführliches und umstrittenes Thema. Eine Autorin, die sich die Frage der neuen Technologien beim Übersetzen stellt, ist Lacommare (2005). In ihrem Beitrag fragte sie sich, ob die neuen Technologien die wahre Zukunft des Übersetzungsberufes sind. Sie definiert, welche technologischen Tendenzen es auf dem Übersetzungsmarkt gibt. Zu guter Letzt zeigt sie, was die neuen Technologien erreichen können und wo ihre Beschränkungen liegen. So beendet sie ihren Beitrag:

„Le Cat (Computer Aided Translation) mira ad affinare i suoi programmi per individuare meglio le analogie. Anche il motore di ricerca Google sta introducendo nuove coppie di lingue per la traduzione automativa. Ma le figure retoriche dovranno ancora essere tradotte dall'uomo."

Dieses Phänomen zeigt sich auch an einem der Produkte, das in Krisenzeiten in Spanien am meisten verkauft werden:. die Smartphones (El País, 2013). Nach Angaben dieser spanischen 
Zeitung, "España es el país de los grandes de la UE que mayor pro. porción de smartphones tiene en Europa.", ist Spanien das Land der EU, das die meisten Smartphones in Europa hat. Dazu kommt, dass $63,2 \%$ der Spanier Smartphonebenutzer sind. Spanien wird hier gefolgt vom Vereinigten Königreich $(62,3 \%)$, Frankreich $(51,4 \%)$, Italien $(51,2 \%)$ und Deutschland $(48,4 \%)$. Im Folgenden betrachten wir, wie Smartphones einen Einfluss auf die Übersetzungs- und Dolmetschtätigkeit gehabt haben.

\section{DIE NEUE AUTOMATISCHEN ÜBERSETZER: ANDROID}

Im App Market wird die Zahl der automatischen Übersetzer ständig erweitert. Dies erklärt sich daraus, dass die Studenten, die eine neue Fremdsprache lernen, sie häufig benutzen. In diesem Abschnitt werden die neuen automatischen Übersetzer und vor allem, die "Übersetzer mit Sprachausgabe" beschrieben. Bei unserer Untersuchung ist dieser Fachausdruck problematisch gewesen, weil es noch keinen offiziellen Ausdruck für diesen App-Typ gibt. Somit mussten wir eine Entscheidung treffen und haben folgende Suchen im Internet bei Google Deutschland ausgeführt:

\begin{tabular}{|c|c|c|c|}
\hline „Übersetzer mit & $\rightarrow$ & 32.600 & Ergebnisse \\
\hline - "Übersetzer mit Stimme" & & 12.900 & $\mathrm{Er}$ \\
\hline - "Sprechende Übersetzer" & $\rightarrow$ & 4.530 & Ergebnisse \\
\hline - "Stimme Übersetzer" & 1 & 2.040 & Ergebnisse \\
\hline "Mündliche Übersetzer". & & 133 & Ergebnisse \\
\hline
\end{tabular}

Unter "Übersetzer mit Sprachausgabe" verstehen wir einen Typ von App, der nicht nur Texteingabe übersetzt, sondern auch Spracheingaben erkennen und übersetzen kann.

Automatische Übersetzer haben sich zu einem praktischen und immer leistungsfähigeren Tool entwickelt, das immer mehr 
Sprachkombinationen besitzt. Heutzutage sind die Übersetzer mit Sprachausgabe eine wichtige Neuigkeit. Tatsächlich sind sie so modern, dass sie noch keinen offiziellen Namen erhalten haben. Diese Apps funktionieren mit einem Spracherkennungssystem. Unter diesen Übersetzern finden wir zwei Typen: 1) jene, die die Aussprache der Texte und Wörter bieten und 2) jene, die wirklich Stimmen und Sprachen erkennen. Diese sind ein Tool, das viel Bezug zum Dolmetschen hat. Wir werden uns auf diese Übersetzer mit Stimme konzentrieren, obwohl wir uns auch den neuen automatischen Übersetzern widmen werden.

In unserer Studie werden wir Android Apps analysieren. Dazu haben wir die zwei Haupmärkte der Android Apps, Samsung Apps und Play Store (auch bekannt als Google Play) untersucht, da in Spanien sind Android-Smartphones wegen der Krise Verkaufschlager sind. Einige Quellen bestätigen, dass in den letzten Monaten die meistverkauften Smartphones in Spanien Android-Smarphones sind (24 horas, 2013).

\subsection{SAMSUNG APPS MARKT}

In unserer Untersuchung haben wir ein Android-Smartphone benutzt. An erster Stelle haben wir den Samsung Apps Markt analysiert, auf dem wir vier verschiedene Suchen ausgeführt haben, die Aspekte wie die Sprache (Spanisch, Deutsch und Englisch) und den Typ der App (Automatischen Übersetzer und Übersetzer mit Sprachausgabe) berücksichtigt haben. Die Suche auf Spanisch "Traductor + voz" hat zu 22 Ergebnissen geführt, wie wir in der folgenden Tabelle sehen können. Diese sind allgemeine Ergebnisse, denn es gibt unter ihnen verschiedene Typen von Apps wie Wörterbücherund automatische Übersetzer, aber kaum Übersetzer mit Sprachausgabe. 
7-in-1 Traductor Offline

Diccionario Ingles Español/English

Spanish Dictionary

El Traductor de viaje

Ficil traductor de idionas

Inglés Traductor

Kategorie

Name des Apps

55 lengutas traductor

Bildung/E-Book

Bildung/E-Book

Nachschlagewerke

$$
\begin{aligned}
& \text { Preis } \\
& \text { Gratis } \\
& 2,88 \mathrm{C}
\end{aligned}
$$

is ayHello Connuricador

iSayHello Conutnicador Pro

Lyngo Lite

Bildung/E-Book

Reisen

Bildung/E-Book

Reisen

Gratis

$7,81 €$

Gratis

Gratis

Gratis

Reisen

Reisen Gratis

$7,00 €$

thai Inglés traductor

Hilfsprogramme

Gratis

Sprechenden Übersetzer

Reisen

Toque Traductor

Bildung/E-Book

$1,99 €$

Traductor de coreano

Bildung/E-Book

Gratis

Traductor de francés

Bildung/E-Book

Gratis

Traductor de Google

Bildung/E-Book

Gratis

Traductor de idiomas

Bildung/E-Book

Gratis

Traductor Inglés

Bildung/E-Book

Gratis

Traductor MacDroid

Bildung/E-Book

Gratis

Traductor que habla

Reisen

Gratis

$1,99 €$ 


\begin{tabular}{|c|c|c|}
\hline Traductor Speak and Translate Free & Reisen & Gratis \\
\hline Traductor Speak and Translate Pro & Reisen & $1,06 €$ \\
\hline Transzilla & Bildung/E-Book & Gratis \\
\hline Trippo Mondo - Traductor Audio & Bildung/E-Book & Gratis \\
\hline Ubersetzer SET German FREE & Reisen & Gratis \\
\hline Wörterbuch (by Farlex) & Nachschlagewerke & Gratis \\
\hline $\begin{array}{l}\text { Wörterbuch Englisch-Deutsch/German- } \\
\text { English }\end{array}$ & Bildung/E-Book & Gratis \\
\hline
\end{tabular}

Abbildung 1. Mira Rueda, C. Übersetzungapps in Samsung Apps

(Stand: 10.12.2013).

Wenn wir das Wort "Übersetzer" auf Deutsch suchen, erzielen wir keine Ergebnisse. Dies könnte am Gebrauch eines spanischen Android-Smartphones liegen, obgleich wir zahlreiche Ergebnisse erhalten, wenn wir "Translator" (233 Ergebnisse) und "Voice + translator" (710 Ergebnisse) suchen. Wie es uns zuvor mit der Suche auf Spanisch passiert ist, fallen diese Apps ganz unterschiedlich aus. Unter diesen Ergebnisse haben wir Kurse, Wörterbücher, usw. erzielt. Unter allen diesen Ergebnissenmöchten wir vor allem eine kostenlose Anwendung hervorheben. Diese heißt "Photo Translator Free“ (Bildung/E-Book). Aber wie funktioniert sie? Da müssen wir Fotos machen, und die App erkennt dann den Text dieses Fotos und übersetzt ihn.

Unter unseren Ergebnissen haben wir auch anekdotische Entdeckungen gemacht. Wir haben zwei Apps gefunden, die Tiersprachen und Menschen-Katzensprache übersetzen. Es sind "Animal Translator" (Samsung Apps) und "Traductor Humano-Gato" (Google PLAY). Schließlich empfehlen wir unter allen kostenlosen automatischen Übersetzern unter den Samsung Apps die folgenden: 
- "Hablando Traductor/Diccionario" oder "Talking Translator/Dictionary" (Bücher und Nachschlagewerke).

- "Traductor de Google" oder "Google Übersetzer" (Business/ Nachschlagewerke).

- "WinTranslator Traductor" (Produktivităt/Business).

- "Photo Translator Free" (Reisen).

\subsection{PLAY STORE MARKT}

An zweiter Stelle haben wir Play Store Markt (oder Google Play) analysiert. Die folgenden Apps, die wir in der nächsten Tabelle aufstellen, sind gratis. Die Mehrheit dieser Apps haben ein Spracherkennungssystem:

\begin{tabular}{ll}
\hline \multicolumn{1}{c}{ Name des Apps } & \multicolumn{1}{c}{ Name des Entwicklers } \\
\hline 55 Lenguas traductor & ShaafStudio \\
\hline 7-in-1 Traductor Offline & SkyCode UK Limited \\
\hline Aion Traductor & Vayzor \\
\hline All ranslate & Michael Spoida \\
\hline Android Traductor & mobeela.com \\
\hline Discurso al Texto Traducir & FSM Software \\
\hline El intérprete - traductor free & Dolmen App Software - by Vincenzo \\
\hline El traductor de Twitter & Reaching High \\
\hline El traductor de viaje & Jourist Verlags GmBH \\
\hline El traductor de viaje Lite & Jourist Verlags GmBH \\
\hline
\end{tabular}




\begin{tabular}{|c|c|}
\hline El tridtuctor que liabla & Revive Apps \\
\hline Fícil traductor de idiomas & NyxCore \\
\hline GTrainslate traductor & CMobile \\
\hline Hablando Traductor/Diccionario & GreenLife Apps \\
\hline Hola traducción & Martin Kim \\
\hline Image it - turísmo traductor & Nicolas Campourcy \\
\hline Inglés traductor & Pleng Apps \\
\hline Inglés-Español Traductor & IDEOAPPS \\
\hline Instant Traductor & FlyJam \\
\hline Intérprete de voz - Traductor & A Digital Tree \\
\hline iTranslate & Sonico Mobile \\
\hline Jibbigo Traductor & Jibbigo \\
\hline Lyugo + traductor de voz & Radish apps \\
\hline Multi Traductor con Voz & Luis Miguel F. P. \\
\hline PAL 9000: Traductor & Daniel Hammeley \\
\hline Skyrim Traductor & Gualberto Soria \\
\hline SMS Traductor & MENA LABz \\
\hline Talk-a-Droid Pro Traductor & Ideele \\
\hline Traducción de idiomas & Hammy apps \\
\hline Traducción en línea & Sapir Benita Shelly \\
\hline Traducción gratis en linea & Adcoms \\
\hline Traducción instantánea & devpenta \\
\hline
\end{tabular}

Futhark 9 (2014)

ISSN 1886-9300

Mira, Der Übersetzungssektor, 285-310 


\section{8}

Traincir libre

Traducir mis palabras

Traincir woz

Traductor

Traductor

Traductor

Traductor de Google

Traductor de idiomas

Traductor de idiomas milltiple

Traductor an varios idiomas

Traductor Europea

Traductor gratis

Traductor gratis

Traductor Hábil

Traductor Keval Soft

Traductor MacDroid

Traductor Multilenguaje

Traductor Multilingue

Traductor múltiples ventanas

Traductor para conversaciones

Traductor por UC Browser

Traductor PRO

Planetinpocket

Voice Translate

Recommended!

3 Loops

AndLabs

Google Inc.

GK Apps

NyxCore

Team Simple

GK Apps

Jeffrey Rosko

Berrie Cool Designs

Emerald Stream

Keval Soft

MacDroid v2

Educational Apps Free

translator

Multi-Window Platform for Android

NyxCore

UCWeb Inc 


\begin{tabular}{|ll|}
\hline Traductor que liabla & Jeffrey Rosko \\
\hline Traductor Speak \& Translate & Pavel Donov \\
\hline Traductor TrausZilla Pro & Borixo \\
\hline Traductor Universal & Jose M. Gomez \\
\hline TransUp Lite Traductor & sonneset \\
\hline TransUp Traductor & sonneset \\
\hline TransZilla traductor & Borixo \\
\hline Trippo Mondo - Traductor & Cellictica Ltd. \\
\hline TS Traductor & Time Space System Co., Ltd. \\
\hline TS Traductor [10 idiomas] & Time Space System Co., Ltd. \\
\hline Vocre Traducir & Vocre \\
\hline Voice Translator & axis \\
\hline Voice Translator Free & Navee Technologies \\
\hline Voice Translator Free & Smart Mobile Software \\
\hline Yak Traductor de Idiomas & World Concepts, Inc. \\
\hline
\end{tabular}

Abbildung 2. Mira Rueda, C. Übersetzungapps im Play Store/Google Play (Stand: 10.12.2013). 


\subsection{VERGLEICHENDE BEWERTUNG DER APPS}

Wir haben drei automatische Übersetzer empfohlen: Talking Translator/Dictionary, Traductor de Google, WinTranslator traductor und Photo Translator Free.

In diesem Abschnitt bieten wir einen Überblick über vier der berühmten Apps, die kostenlos sind.

3.3.1 "HABLANDO TRADUCTOR/DICCIONARIO ${ }^{\prime \prime}$ ODER "TALKING TRANSLATOR/DICTIONARY" (BÜCHER UND NACHSCHLAGEWERKE)

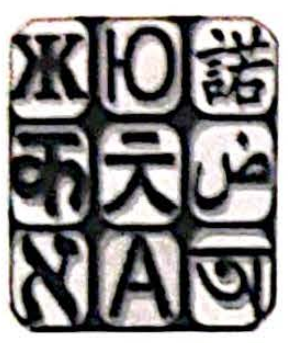

a) Wenn wir etwas sagen und übersetzen (ohne $z u$ schreiben, das heißt, per Spracheingabe) möchten, erkennt die App unsere Stimme, aber nicht die Sprache, die wir sprechen. Zuvor müssen wir das Sprachenpaar wählen.

b) Wenn wir unsere Stimme benutzen um zu übersetzen, spricht die App die Äquivalenten oder den übersetzten Text aus (Sprachausgabe).

c) Diese App enthält ein Wörterbuch, einen Suchlauf und das Wort des Tages.

d) Die volle App ist noch nicht übersetzt. Es gibt noch verschiedene Informationen auf Englisch, wie die Liste der Sprachen.

e) Sie enthält auch Satzkorrektur. Wenn wir zum Beispiel schreiben: „Ich weiss es nuicht.“, fragt die 
App uns: „Möchten Sie sagen: ich weiss es nicht? Ja oder nein?"

f) Sie übersetzt gesprochene und getippte Texte. Sie erkennt keine handschriftlichen Texte.

g) Diese App unterstützt 81 Sprachen, die geografisch (und auf Englisch) gegliedert sind:

- African (Afrikaans, Hausa, Igbo, Somali, Swahili, Yoruba and Zulu),

- Asian-East (Cebuano, Chinese, Filipino, Hmong, Indonesian, Japanese, Javanese, Khmer, Korean, Lao, Mongolian, Thai and Vietnamese),

- Asian-South/Indian (Bengali, Gujarati, Hindi, Kannada, Malay, Marathi, Nepali, Punjabi, Tamil, Telugu and Urdu),

- Asian-West (Arabic, Armenian, Azerbaijani, Georgian, Persian and Turkish),

- European (Albanian, Basque, English, Estonian, Finnish, Greek, Hebrew, Hungarian, Latvian, Lithuanian, Maltese, Polish, Serbian and Slovenian),

- European-Celtic (Irish and Welsh),

- European-Germanic (Danish, Dutch, German, Icelandic, Norwegian, Swedish and Yiddish),

- European-Romance (Catalan, French, Galician, Italian, Latin, Portuguese, Romanian and Spanish), 
- European-Slavic (Belarusian, Bosnian, Bulgarian, Croatian, Czech, Macedonian, Russian, Slovak and Ukrainian),

- More (Esperanto, Haitian Creole and Maori).

3.3.2 "TRADUCTOR DE GOOGLE" ODER "GOOGLE ÜBERSETZER" (BUSINESS/ NACHSCHLAGEWERKE/TOOLS)

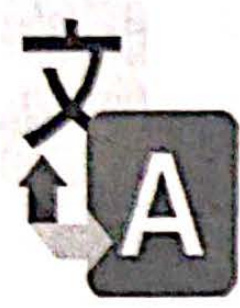

a) Wenn wir etwas sagen und übersetzen (ohne $\mathrm{zu}$ schreiben, das heißt, per Spracheingabe) möchten, erkennt die App unsere Stimme, aber nicht die Sprache, die wir sprechen. Zuvor müssen wir das Sprachenpaar wählen.

b) Wenn wir unsere Stimme benutzen um $z u$ übersetzen, spricht die App die Äquivalenten oder den übersetzten Text aus (Sprachausgabe).

c) Diese App enthält ein Wörterbuch und einen Suchlauf.

d) Sie kann unsere SMS übersetzen.

e) Sie erkennt auch Text in Fotos und übersetzt ihn, aber vorher müssen wir das Sprachenpaar wählen.

f) Sie enthält auch Satzkorrektur. Wenn wir schreiben: "ich haisse paul.", fragt die App uns: Möchten Sie sagen: „ich heisse paul." ?

g) Diese App unterstützt 84 Sprachen. 
h) Sie ubersetzt gesprochene, getippte und handschriftliche Texte.

i) Sie kann auch offline übersetzen.

\subsection{3 "WINTRANSLATOR TRADUCTOR“ (PRODUK- TIVITÄT/BUSINESS)}

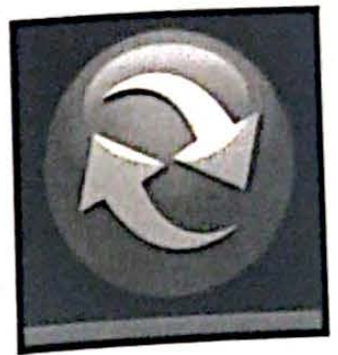

a) Wenn wir etwas sagen und übersetzen (ohne zu schreiben, das heißt, per Spracheingabe) möchten, erkennt die App unsere Stimme, aber nicht die Sprache, die wir sprechen. Zuvor müssen wir das Sprachenpaar wählen.

b)

Wenn wir unsere Stimme benutzen um zu übersetzen, spricht die App nicht die Äquivalenten oder den übersetzten Text aus (keine Sprachausgabe).

c)

Sie enthält auch keine Satzkorrektur. Wenn wir schreiben: „Ich haisse paul.“ ,übersetzt die App den Satz. Das Ergebnis, das wir erzielt haben, ist: „Me haisse paul."

d)

Diese App unterstützt 63 Sprachen.

e)

Sie übersetzt gesprochene und getippte Texte. 


\subsection{4 "PHOTO TRANSLATOR FREE“ (REISEN).}

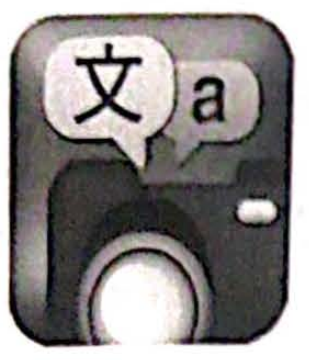

a) Wenn wir etwas übersetzen möchtebn (ohne zu schreiben), erkennt die App nicht unsere Stimme (keine Spracheingabe).

b) Hier ist es nicht möglich, unsere Stimme zu benutzen um zu übersetzen, weil diese App keine Sprachausgabe hat.

c) Die volle App ist noch nicht übersetzt. Es gibt noch verschiedene Informationen auf Englisch, wie die Liste der Sprachen.

d) Sie enthält keine Satzkorrektur.

e) Sie übersetzt nur Texte, die auf Fotos zu sehen sind.

f) Die kostenlose Version dieser App unterstützt 5 Sprachen:

- Deutsch

- Englisch

- Französisch

- Spanisch

- Thai

(*) Die volle Version unterstützt mehr als 25 Sprachen. 
Diese Apps werden immer perfekter und weiterentwickelter. Die neuen Übersetzer mit Sprachausgabe sind tatsächlich ein Beispiel für eine Annäherung ans Dolmetschen.

Im Dolmetschunterricht könnten wir unseren Studenten diese Apps vorstellen, da das Arbeiten mit diesen Tools sehr interessant sein kann. Alle Lehrkräfte wissen, dass die Studenten heimlich automatische Übersetzer benutzen, vor allem, wenn sie Aufsätze, Übungen oder Übersetzungen machen müssen. Obwohl wir ihnen immer empfehlen, keine automatischen Übersetzer benutzen, können wir sie die Erfahrung machen lassen. Wenn sie verschiedene Übungen machen, können sie herausfinden, ob die automatischen Übersetzer und Übersetzer mit Sprachausgabe gut oder schlecht sind und über diese Erfahrung verstehen, welche positive und negative Aspekte automatische Übersetzer und Übersetzer mit Sprachausgabe haben, und sich vor allem ihre eigene Meinung dazu bilden.

Diese Übersetzer können außerdem nützlich sein. Unsere Studenten können sie benutzen, um eine Übung zur Aussprache zu machen. Sie können zu zweit oder in kleinen Gruppen arbeiten. Mit dieser App können sie sofort wissen, ob sie gut oder schlecht ausgesprochen haben, je nachdem, ob die Übersetzer sie gut oder schlecht verstanden haben.

Mit dieser App können wir auch andere Übungen machen, z. B. die Unterschiede zwischen (einsprachigen und zweisprachigen) Wörterbücher und automatischen Übersetzer suchen. Auf diese Weise können wir Realität in unsere Unterrichtstunde bringen. Ein wichtiger Aspekt, den unsere Studenten lernen können, ist, wie sie das Beste aus diesen, teilweise kostenlosen, Tools herausholen können..

Diese Art des Lernens stellt eine ganz neue Methode dar, die MLearning heißt. Bei diesem didaktischen Konzept handelt es sich darum via mobilen Endgeräten bzw Handys, PDAs oder Tablets, zu lernen. Eine Autorin, die eine Expertin auf dem Gebiet ist, Pouezevara (2012), spricht sich auch für die M-Learning-Methode aus, weil sie den Studenten großen Nutzen bringen kann. Sie meint tatsächlich, dass die mobilen Endgeräte den Studenten Nutzen in ihrem Lernprozess bringen können, und gliedert diese Methode in vier Teile: 
"Accessibility (access to learning opportunities, experts/mentors, other learners); immediacy (on-demand learning, real-time communication and data sharing, situated learning); individualized (bite-size learning on familiar devices; promotes active learning and a more personalized experience); and intelligence (advanced features make learning richer through context-aware features, data capture, multimedia)."

Auf anderen Internetseiten, die von der Entwicklung der Ausbildung (Mobile Learning) handeln, definiert beispielsweise JISC (2011), was Mobile Learning ist, wie es funktioniert und warum es schon eine Tendenz wird:

"Mobile leaming involves change in the sense that the ability to communicate with tutors and peers, as well as access learning resources, changes what is possible in education. It takes initiative for leaders to create a vision to sustain that change and, finally, mobile learning requires adaptability by members of staff to carry out the change."

\section{DIE KRISE IM ÜBERSETZUNGS- UND DOLMETSCHUNTERRICHT}

In diesem Abschnitt werden wir unter einem anderen Gesichtspunkt noch die Krise behandeln. Diesm al werden wir einen Überblick über die aktuelle Lage der Universitätslehre, vor allem über die Lage des Übersetzungs- und Dolmetschunterrichts, in Spanien bieten. Die Krise zeigt sich in unseren Hörsälen auf unterschiedlichste Weise. In den letzten Jahren haben wir zwei verschiedene Tendenzen der Immatrikulationszahlen beobachten können. In einigen Fächer gibt es eine Zunahme der Zahl der immatrikulierten Studenten, die ein sehr definiertes Profil haben. Diese Studenten sind häufig gut ausgebildetund haben zuvor eine Arbeit gehabt. Wegen der Krise haben sie ihren Arbeitsplatz verloren. Also entscheiden sie sich, ihre Ausbildung fortzusetzen. Die Weiterbildung ist für diese Studenten die Chance, eine (hochwertige) Arbeit zu finden. Unter diesen Studenten finden wir aber auch junge Leute, die ihr Studium vor kurzem beendet haben und noch keine Arbeit gefunden haben. Ihre Rückkehr zur Universität bedeutet eine große Herausforderung für 
die Dozenten. Die Mehrheit dieser Studenten bezahlt auf eigene Rechnung ihre Studien und ist normalerweise älter als ihre anderen Kommilitonen. Ihre Erwartungen sind entsprechend sehr hoch und manchmal auch ihre Motivation, aber nicht immer, da diese Studenten sich gezwungen gesehen haben, ihre Ausbildung mangels eines Arbeitsplatzes wieder fortzusetzen.

Es gibt aber auch eine gegenläufige Tendenz bezüglich der Immatrikulationszahlen. Vor allem in diesem Jahr, haben viele Studenten ihr Studium aufgegeben, weil ihnen kein Stipendium gewährt wurde. Leider ist dies kein Einzelfall in Spanien.

Im Fall des Übersetzungs- und Dolmetschunterrichts treffen wir auf eine weitere Schwierigkeit: Übersetzung und Dolmetschen ist ein multidisziplinäres Studium, das ein hohes Sprachniveau erfordert. Daher kann der Mangel an Motivation die Hauptursache des Studienabbruchs sein.

In der aktuellen Krisenzeit und vor diesem Hintergrund, spielen die Übersetzungs- und Dolmetschdozenten dabei eine Hauptrolle. Sie müssen ihre Studenten wie nie zuvor motivieren. Die Priorität der Dozenten muss ihre Studenten sein. Die Dozenten müssen besonders gut überlegen, wie sie ihre Unterrichtstunden planen, um den Erwartungen der Studenten zu entsprechen, und sie gut für den aktuellen Arbeitsmarkt vorzubereiten. ZurMotivation kann die Technologie viel beitragen, aber es sollten immer gute Dozenten ihre Studenten unterrichten, egal wie hochentwickelt die Technologie ist, denn menschliche Übersetzer und Dolmetscher sind noch nicht ersetzbar. Die Kommunikation ist eine menschliche Aktivität, in der die Technologie nur ein Mittel ist, das sie begünstigen kann, aber eben nur ein Mittel.

\section{FAZIT}

Obwohl die Krise eine schwierige Zeit ist, können wir sie benutzen, um neue Wege zu finden. Die finanzielle Krise hat auch den Übersetzungs- und Dolmetschsektor beeinflusst. Wegen der Krise werden bestimmte Produkte, die nützlich sind, wie Handys, Software 
und Apps, besonders viel gekauft.. Solche Apps wie automatische Übersetzer und Übersetzer mit Sprachausgabe, die wir analysiert haben, sind ein eindeutiges Beispiel für Innovation, technologische Entwicklung und Kreativität.

Die Übersetzer und Dolmetscher sind sachkundige Spezialisten, die eine gute berufliche Vorbereitung erhalten, um Widrigkeiten trotzen zu können. Überdies ist die Anpassungsfähigkeit der Übersetzer und Dolmetschen ein positiver Faktor auf dem Arbeitsmarkt. Einer der Faktoren, der heutzutage mehr Einfluss hat, ist die Kreativität, denn sie macht den Unterschied. Deshalb sollen die Dozenten ihre Studenten anregen, die NIKT (neue Informations- und Kommunikationstechnologien) nicht nur in ihrem Studium, sondern auch in ihrer berufliche Zukunft zu verwenden. In diesem Bereich sollte man mehr Kurse, Kongresse und Seminare bieten zu den Themen: 1) Wie man bessere Sichtbarkeit im Internet haben und 2) Wo kann man die Weiterausbildung fortsetzen kann. Das kann sehr interessant und wertvoll für die berufliche Zukunft sein. Der aktuelle Arbeitsmarkt ist sehr wettbewerbsintensiv. Das ist der Grund, weshalb man kompetente, kreative und höchst ausgebildete Spezialisten sucht. Es wäre auch sehr praktisch für unsere Studenten zu wissen, wie sie die NIKT, offene Internetkurse (MOOCs), u.a. nutzen können. Im Internet gibt es verschiedene, sehr nützliche Ressourcen für diejenigen, die vor einiger Zeit ihr Studium beendet haben, und die ihre Kenntnisse auffrischen möchten. Zur Förderung der Allgemeinbildung gibt es immer mehr kostenlose offene Internetkurse, die meistenvon ihnen von renommierten Universitäten angeboten. Das ist eine gute Art, unsere Weiterbildung fortzusetzen, ohne extra Ausgaben machen zu müssen. 


\section{BIBLIOGRAFISCHE REFERENZEN}

"Cómo huir del desánimo colectivo en el trabajo", Cincodías, 1. März 2013. Web, <http://tinyurl.com/kooq413> (Letzter Zugriff am: 5. August 2013). „La crisis no puede con los buenos traductores”, La Razón, 16. Mai 2012. Web, $<$ http://tinyurl.com/o8s89a3> (Letzter Zugriff am: 5. August 2013).

"Los autónomos dados de alta en Seguridad Social crecer por primera vez desde 2009", Cincodias, 5. Juni 2013. Web, <http://tinyurl.com/k5znzpd> (Letzter Zugriff am: 5. August 2013).

"Aplicaciones", Google Play, 07. August 2013. Web, <https://play.google.com/intl/es_es/about/apps/> (Letzer Zugriff am: 10. Dezember 2013).

„El ,smartphone' vence a la crisis”, El País, 10. Januar 2013. Web, <http://tinyurl.com/9wl9dkb> (Letzter Zugriff am: 6. August 2013).

„Las ventas de Samsung en España baten récords en plena crisis por el Galaxy", 24 Horas, 16. Juli 2013. Web, <http://tinyurl.com/od96nlg> (Letzter Zugriff am: 6. August 2013).

„Mobile Learning", JISC infoNet, 2011. Web, <http://tinyurl.com/pbjuecb> (Letzter Zugriff am: 10. November 2013).

„Samsung Apps", Samsung Deutschland, 7. August 2013. Web, <http://apps.samsung.com/earth/main/getMain.as> (Letzter Zugriff am: 7. August 2013).

„Samsung Apps", Samsung España, 7. August 2013. Web, $<$ http:// tinyurl.com/16u7wz8> (Letzter Zugriff am: 7. August 2013).

DAvico, Gianni, "Gabriella Gentile, Il mercato delle traduzioni: crisi o declino?", Brainfood: L'industrial della traduzione (e la vita), secondo me. Visioni, previsioni e travisamenti, 21. Januar 2013. Web, $<$ http://tinyurl.com/nznfqpo> (Letzter Zugriff am: 10. November 2013).

GARCíA LUQUE, Francisca, „La palabra crisis en la prensa: análisis terminológico de cara a la enseñanza y al ejercicio profesional de la traducción socioeconómica (francés-español)", Anales de Filología Francesa 18, 2010, p. 203215.

GRELIER, Pauline, „U.E.: La traduction face à l'économie de marche”", Cafébabel 2013. Web, <http://tinyurl.com/khup9ot> (Letzter Zugriff am: 10. November 2013).

HERMIDA, Ana, „Entrevista a Anabel Galán Mañas, profesora de Traducción e Interpretación en la Universitat Autònoma de Barcelona", La linterna del Traductor. La revista multilingüe de Asetrad, 8. Juni 2013. Web, $<$ http:// tinyurl.com/q44z7o7> (Letzter Zugriff am: 10. September 2013).

LACOMMARE, Ilaria, "Traduzione e tecnologia: progresso o tradimento?", Cafébabel 2005. Web, <http://tinyurl.com/qbagwgb> (Letzter Zugriff am: 10. November 2013). 


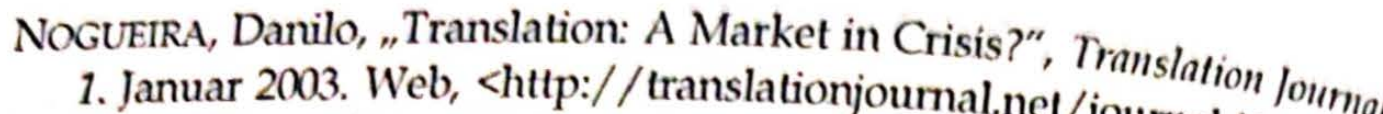
(Letzter Zugriff am: 9. Juli 2013). POUEZEVARA, Sarah, „Mobiles for Teaching and Learning: Trans into Practice", Educational Teclnology Debate. Exploring ICT and Ing Theory Developing Countries. November 2012. Learning in <http://tinyurl.com/qemueb5> (Letzter Zugriff am: 10. November 2013), 